\title{
Summary of the Neutrino Physics session
}

\author{
Rupert Leitner ${ }^{1}$ \\ Charles University in Prague, \\ V Holešovičkách 2, Praha 8, Czech Republic \\ E-mail: Rupert. Leitnerecern.ch
}

\begin{abstract}
Short summary of major results reported at the XIth International Conference on Heavy Quarks and Leptons (HQL2012) is given. These results comprise first measurements of $\sin ^{2} 2 \theta_{13}$, updated results of other oscillation experiments, first measurement of pep and precise measurement of ${ }^{7} \mathrm{Be}$ and Sun neutrinos fluxes, current status and future plans of detection of very high energy neutrinos, summary of searches for neutrino-less double beta decay, evidences for possible existence of light sterile neutrinos and recent measurements of geo-neutrinos.
\end{abstract}

The XIth International Conference on Heavy Quarks and Leptons, Prague, Czech Republic

June 11-15, 2012

$1 \quad$ Speaker 


\section{Introduction}

All neutrino physics reports [1-7] at HQL2012 conference profited from an up to date results that have been recently given at the Neutrino 2012 conference in Kyoto. Only short summary of major results is given below, the details and all relevant references can be found in the talks [1-7].

\section{Measurement of neutrino oscillation parameters}

In three neutrinos flavor frame the neutrino oscillations are described by three mixing angles $\theta_{12}, \theta_{23}$ and $\theta_{13}$, two differences of mass squares $\Delta \mathrm{m}_{21}^{2}$ and $\Delta \mathrm{m}^{2}{ }_{32}$ and one CP violating phase $\delta$. The phase becomes physical parameter only if all the three mixing angles are different from zero. The values of two mixing angles $\theta_{12}, \theta_{23}$ and both mass differences has been precisely measured during past years.

Major breakthrough in neutrino physics in 2012 was the discovery and very precise measurement of non zero value of the smallest mixing angle $\theta_{13}$ in reactor neutrino experiments Daya Bay and RENO and $3 \sigma$ evidence found by Double Chooz experiment. Details of reactor neutrino results have been given in the talk of $H$. Band [1]. Measured values of $\sin ^{2} 2 \theta_{13}$ are summarized in Table 1. Relatively large value of $\sin ^{2} 2 \theta_{13}$ could make it easier to measure direct effects of CP violation in lepton sector of the Standard Model.

\begin{tabular}{|c|c|c|c|}
\hline & Daya Bay & RENO & Double Chooz \\
\hline $\sin ^{2} 2 \theta_{13}$ & $0.089 \pm 0.010 \pm 0.005$ & $0.113 \pm 0.013 \pm 0.019$ & $0.109 \pm 0.030 \pm 0.025$ \\
\hline
\end{tabular}

Table 1: Values of $\sin ^{2} 2 \theta_{13}$ measured by three reactor experiments. First and second uncertainties are statistical and systematic uncertainties respectively.

Indications of non-zero values of $\sin ^{2} 2 \theta_{13}$ have also been found in electron neutrinos appearance in accelerator muon neutrinos beam in T2K and MINOS experiments and were part of the talk of R. Nichol [2]. These results are complementary to results of reactor experiments because they are sensitive to the value of the $\mathrm{CP}$ violating phase $\delta$. Moreover due to the matter effect on propagation of neutrino beam through the rock the results depend also on the sign of $\Delta \mathrm{m}_{32}^{2}$ (so called neutrino hierarchy), see Table 2.

\begin{tabular}{|c|c|c|}
\hline $\sin ^{2} 2 \theta_{13}$ & T2K & MINOS \\
\hline$\delta=0 \Delta \mathrm{m}_{32}^{2}>0$ & $0.104+0.060-0.045$ & $0.01 \div 0.1290 \%$ C.L. \\
\hline$\delta=0 \Delta \mathrm{m}_{32}^{2}>0$ & $0.128+0.070-0.055$ & $0.03 \div 0.1990 \%$ C.L. \\
\hline
\end{tabular}

Table 2: Values of $\sin ^{2} 2 \theta_{13}$ measured by two accelerator experiments.

Measurements of accelerator and atmospheric neutrino disappearance in MINOS, Super-Kamiokande and $\mathrm{T} 2 \mathrm{~K}$ experiments are sensitive to the values $\theta_{23}$ and $\Delta \mathrm{m}^{2}{ }_{23}$. New measurements for the first time suggest non-maximal value of the value of $\sin ^{2} 2 \theta_{23} \neq 1$ [2]. Disappearance of atmospheric neutrinos has been observed also in ANTARES experiment; see 
the talk of J. Brunner [3] where current status of atmospheric and cosmic neutrinos with very high energies is given.

New event of tau neutrino interaction has been found in the data collected by OPERA experiment, Super-Kamiokande experiment observed an excess of events that could be caused by tau neutrinos.

\section{Sun neutrinos and MSW}

The most recent data from Borexino, SNO and Super-Kamiokande have been reported by F. Rannuci [4].

Borexino experiment has provided the first measurement of solar pep and precise measurement of ${ }^{7} \mathrm{Be}$ monoenergetic neutrino fluxes. Neutrino energies from ${ }^{7} \mathrm{Be}$ and pep are in the region where predicted survival probability changes due to MSW effect. These measurements are therefore very important in order to confirm MSW effect on neutrino passage through the Sun matter. An interesting result has been found by re-analysis of ${ }^{8} \mathrm{~B}$ fluxes by SNO experiment that indicated little discrepancy with the prediction in 4-9 MeV energy region.

\section{Majorana or Dirac neutrinos}

Neutrinos are the only neutral elementary fermions and could be either of Dirac or Majorana nature. In latter case, processes that violate lepton number by two units would be possible. Among them the best experimental limits are for so called neutrinoless double beta decays ${ }^{\mathrm{A}} \mathrm{Z} \rightarrow{ }^{\mathrm{A}}(\mathrm{Z}+2)+2 \mathrm{e}^{-}$of some even-even isotopes. Neutrino-less double beta decay rate depends on the value of absolute effective mass of electron neutrinos. Observation and precise measurements of neutrino-less double beta decays will therefore not only reveal Majorana origin of electron neutrinos but also will determine the absolute scale of neutrino masses that is not accessible by neutrino oscillation experiments.

Overview of current experimental status has been given in the talk of O.Cremonesi [5] published in these proceedings. The best limits for neutrino masses are $<0.44,0.6$ and $0.3 \mathrm{eV}$ from experiments with ${ }^{76} \mathrm{Ge},{ }^{130} \mathrm{Te}$ and ${ }^{136} \mathrm{Xe}$ isotopes respectively. In case of ${ }^{76} \mathrm{Ge}$ there is one positive claim suggesting the value of neutrino mass to be $0.32 \pm 0.03 \mathrm{eV}$. Confirmation of rejection of that result is expected to happen in near future.

Sensitivity of future experiments is planned to be very close to or even better than the lowest possible values of electron neutrino masses $\sim 0.05 \mathrm{eV}$ expected for so called inverted hierarchy of neutrino masses with an ordering $\mathrm{m}_{3}<\mathrm{m}_{1}<\mathrm{m}_{2}$ of masses of neutrinos eigenstates.

\section{Light sterile neutrinos}

From the LEP measurements of $\mathrm{Z}$ boson line shape it is known that there are only three families of light neutrinos that interact with the Z. However there are several hints that might be explained by the existence of light sterile neutrino specie(s): LSND anomaly, MiniBoone anti-neutrino results, $\mathrm{Ga}$ and reactor anomalies, cosmological determination of number of neutrinos species. J. Link [6] gave an overview of current 
experimental status and plans to address possible existence of sterile neutrino(s) with masses in $1 \mathrm{eV}$ region.

\section{Geo-neutrinos}

Currently two experiments Borexino and KamLand measured electron antineutrinos from beta decays of isotopes in Earth core. Talk of S. Zavatarelli [7] gave present status and future plans of this very new interdisciplinary field. Both experiments observed geo-neutrinos signal at $4 \sigma$ level. Although statistically limited, the results of Borexino and Kamland indicate contribution to neutrino flux from the Earth mantle. More precise measurements are expected by Kamland with reactors switched off, in near future there will be more data taken by $\mathrm{SNO}+$ experiment.

\section{References}

[1] H. Band, on behalf of the Daya Bay Collaboration, Observation of Nonzero $\theta 13$ at Reactor Antineutrino Experiments, talk given at HQL2012, Prague, June 2012, proceedings entry: http://pos.sissa.it/archive/conferences/166/051/HQL\%202012 051.pdf

[2] R. Nichol, Long baseline Neutrino Oscillation Experiments, talk given at HQL2012, Prague, June 2012.

[3] J. Brunner, High energy neutrinos, talk given at HQL2012, Prague, June 2012.

[4] F. Rannuci et al. (Borexino Collaboration), Recent Results on Solar Neutrinos, talk given at HQL2012, Prague, June 2012, proceedings entry: http://pos.sissa.it/archive/conferences/166/052/HQL\%202012_052.pdf

[5] O.Cremonesi, Developments on double beta decay search, talk given at HQL2012, Prague, June 2012, proceedings entry: http://pos.sissa.it/archive/conferences/166/054/HQL\%202012_054.pdf

[6] J. Link, Light Sterile Neutrinos: The Evidence, talk given at HQL2012, Prague, June 2012.

[7] S. Zavatarelli, The study of geo-neutrinos, talk given at this HQL2012, Prague, June 2012, proceedings entry: http://pos.sissa.it/archive/conferences/166/053/HQL\%202012_053.pdf 